\title{
Growth response of Oryza sativa seedlings to graphene oxide and its variability among genotypes
}

\author{
Y. HE ${ }^{1,+}$, H.M. WEI ${ }^{1,+}$, S.J. LIU ${ }^{1}$, Y.C. XU ${ }^{1}$, Z.Y. ZHU ${ }^{1,2}$, H. YAN ${ }^{1}$, J.X. LI ${ }^{1,2, *}$, and Z.H. TIAN ${ }^{1,2, *}$ \\ ${ }^{1}$ College of Life Science, Yangtze University, Jingzhou 434025, P.R. China \\ ${ }^{2}$ South China Botanical Garden, Chinese Academy of Sciences, Guangzhou 510650, P.R. China \\ *Corresponding authors: E-mail: jxli@scbg.ac.cn, zhtian@yangtzeu.edu.cn
}

\begin{abstract}
With the extensive utilization of graphene nanomaterials, they inevitably enter our environment. The potential phytotoxicity and environmental impact of graphene oxide (GO) have recently attracted much attention. We designed the experiment based on seed germination, seedling morphology, physio-biochemical properties, and antioxidant enzyme activities of five rice genotypes (9311, MH63, R527, K866, and Nipponbare) under six concentrations of GO (0, 5, 10, 50,100 , and $150 \mathrm{mg} \mathrm{dm}^{-3}$ ). We studied the effects of different concentrations of GO on germination index (GI), shoot length (SL) and root length (RL), adventitious root number, shoot and root fresh masses, root/shoot ratio, chlorophyll (Chl) content, malondialdehyde content, and activities of superoxide dismutase (SOD), catalase (CAT), and peroxidase (POD). Graphene oxide treatments significantly enhanced seed germination and root growth and inhibited shoot growth of all genotypes. Furthermore, we found a significant genotype-dependent response to GO treatments. According to the relative increment trend of GI, SL, and RL, root/shoot ratio, antioxidant enzyme activities (CAT, POD, and SOD), and Chl content, 'R527' showed more tolerance to GO treatments than the other four genotypes. The 'MH63' and 'K866' were more sensitive than 'Nipponbare' and '9311'. It indicates that the GO-tolerant genotype might avoid free radicals damage from GO by increased antioxidant enzyme activities. Moreover, we should consider the genotype differences when evaluating the potential phytotoxicity of GO and environmental risk to ecosystems.

Keywords: antioxidant enzymes activities, chlorophyll content, malondialdehyde, seedling morphology, graphene oxide, Oryza sativa.
\end{abstract}

\section{Introduction}

Graphene-family nanomaterials (GFNs) have been widely employed in manufacturing, electronics, energy, biomedicine, and environment protection due to their high surface area, unique optical properties, good thermal conductivity, excellent electrical conductivity, and a high mechanical strength. With the increasing production and intensive use of GFNs, they inevitably enter the air, water, and soil (Keller and Lazareva 2013). Because of their huge surface area and unique chemical structure, GFNs can be the poisonous and harmful substances in the environment and cause damage to the animals, plants, and microorganisms (Xu et al. 2009). The GFNs could produce different effects on plants such as accumulation in plant organs, antibacterial activity, negative or positive effects on growth, physiological and biochemical traits, and quality of produced food (Liu et al. 2015, Cheng et al. 2016, Jiao et al. 2016a,b, Chen et al. 2017, Zhou and Hu 2017, Hao et al. 2018, Huang et al. 2018). We reported the effects of graphene on germination and seedling morphology of rice (Oryza sativa L. ssp. indica and ssp. japonica, Liu et al. 2015), but we do not know whether the effects are genotype-dependent. Therefore, we designed the

$\overline{\text { Received }} 8$ April 2020, last revision 31 July 2020, accepted 24 August 2020.

Abbreviations: ARN - adventitious root number; CAT - catalase; Chl - chlorophyll; EDTA - ethylenediaminetetraacetic acid; GFNs graphene-family nanomaterials; GI - germination index; GO - graphene oxide; MDA - malondialdehyde; NBT - nitroblue tetrazolium; PBS - phosphate buffer solution; POD - peroxidase; PVP - polyvinylpyrrolidone; RFM - root fresh mass; RL - root length; R/S - root/ shoot ratio; SFM - shoot fresh mass; SL - shoot length; SOD - superoxide dismutase.

Acknowledgements: This work was supported by the Open Project from Guangdong Provincial Key Laboratory of Applied Botany of China (AB2018008) and the Science and Technology Research Project of Educational Commission of Hubei Province of China (B2015450). ${ }^{+}$These authors contributed equally.

Conflict of interest: The authors declare that they have no conflict of interest. 
experiment to investigate the different effects of graphene oxide (GO) on seed germination, seedling morphology, physio-biochemical properties, and antioxidant enzyme activities of five different genotypes of cultivated rice. It will be a benefit to a better understanding of the effects of GFNs on plants and evaluating their environmental risks.

\section{Materials and methods}

Plants and growth conditions: In this study, we employed four indica rice (O. sativa L. ssp. indica) genotypes 9311, MH63, R527, and K866, and one japonica rice (O. sativa L. ssp. japonica) genotype Nipponbare. Seeds of all genotypes were obtained from the College of Life Science, Yangtze University, China. Graphene oxide solution of $3.8 \mathrm{~g} \mathrm{dm}^{-3}$ were purchased from Longliang Biological Technology Co., Nanjing, China. Rice seeds with uniform size were disinfected with $2 \%(\mathrm{~m} / \mathrm{v}) \mathrm{NaClO}$ for $10 \mathrm{~min}$. The sterilized seeds were placed in 9-cm Petri dishes with sterilized double layer filter papers soaked with 5, 10, 50, 100 , and $150 \mathrm{mg} \mathrm{dm}^{-3} \mathrm{GO}$ solutions (diluted with pure water and pure water was used as control) for germination in the dark at $25 \pm 1{ }^{\circ} \mathrm{C}$. All treatments were carried out with 40 seeds in triplicate.

Effects of GO on seed germination and seedling morphology: A seed that its radicle broke through the seed coat and reached at least to the seed length, and its germ was at least half of the seed length, was considered to be germinated (Liu et al. 2015, Tribouillois et al. 2016). The germination index (GI) calculation was conducted according to the method reported by Wang et al. (2016): $\mathrm{GI}=\sum G i / I$, where $G i$ is the number of germination in $I$ day (the time of cultivation).

Twenty-five uniform seedlings were randomly selected, and each seedling was anchored with a sterilized sponge, then transferred into a plastic water bucket containing $200 \mathrm{~cm}^{3}$ of different concentration GO solutions. Three replicates were employed in each treatment. The seedlings were cultivated in plant incubator (HP1500GS-D, Ruihua, China, set to a 14-h photoperiod, an irradiance of $165 \mu \mathrm{mol} \mathrm{m} \mathrm{m}^{-2} \mathrm{~s}^{-1}$, a temperature of $25 \pm 1{ }^{\circ} \mathrm{C}$, and a relative humidity of $60 \%$.

After $16 \mathrm{~d}$, at least ten seedlings were randomly chosen from each replicate. Shoot length (SL), root length (RL), and adventitious root number (ARN) of each seedling were determined. Then, after removed the seed coats and drained the surface water of root, the root and shoot of each seedling were cut from the root base. The shoot fresh mass and root fresh mass were measured and used to calculate the root/shoot ratio $(\mathrm{R} / \mathrm{S})$.

Determination of chlorophyll and malondialdehyde content and antioxidant enzymes activities: To determine the chlorophyll (Chl) $a+b$ content, 0.2-g leaf samples were extracted using $25 \mathrm{~cm}^{3}$ of $80 \%(\mathrm{v} / \mathrm{v})$ acetone and the absorbance of extract was measured at 645 and $663 \mathrm{~nm}$ using a spectrophotometer (UV-2600, Shimadzu, Kyoto, Japan) according to the method described by Arnon
(1949).

Root samples $(0.5 \mathrm{~g})$ were homogenized with $7.5 \mathrm{~cm}^{3}$ of cold phosphate buffer solution (PBS; $\mathrm{pH} 7.8$ ) containing $1 \%(\mathrm{~m} / \mathrm{v})$ polyvinylpyrrolidone (PVP) and $0.2 \mathrm{mM}$ EDTA, and centrifuged at $10625 \mathrm{~g}$ and $4{ }^{\circ} \mathrm{C}$ for $30 \mathrm{~min}$. The supernatants were used to analyse malondialdehyde (MDA) content carried out by the trichloroacetic acid and 2-thiobarbituric acid method (Heath and Packer 1968, Siddiqui et al. 2015a,b).

Root samples $(0.2 \mathrm{~g})$ were used to measure the activities of antioxidant enzymes via the method reported by Cakmak and Marschner (1992). Superoxide dismutase (SOD) activity was determined based on the oxidationreduction reaction of nitroblue tetrazolium (NBT). The SOD activity unit was defined as the amount of enzyme used to inhibit $50 \%$ NBT reduction at $560 \mathrm{~nm}$ (Cakmak and Marschner 1992). Catalase (CAT) activity was measured by monitoring the decomposition of $\mathrm{H}_{2} \mathrm{O}_{2}$ at $240 \mathrm{~nm}$ in the reaction using peroxidase as a catalyst (Abei 1984, Cakmak and Marschner 1992, Siddiqui et al. 2015b). Peroxidase (POD) activity was performed with the method based on guaiacol oxidation reaction and determined by the growth of absorbance at $470 \mathrm{~nm}$ (Cakmak and Marschner 1992) per minute.

Data handling and statistics analysis: All data were described as the means \pm standard deviations. Statistical analysis was performed with a two-way analysis of variance $(A N O V A)$ after homogeneity of variance test with the SPSS v. 17.0 statistical software (SPSS Inc., Chicago, USA). Moreover, Duncan's multiple range tests were employed to compare the significance of means at $P<0.05$ or $P<0.01$.

\section{Results}

In our study, the performance of seed germination was assessed as GI, and seeding morphology was performed with SL, RL, ARN, shoot fresh mass, root fresh mass, and $\mathrm{R} / \mathrm{S}$. The rice genotype-dependent and GO dosedependent effects were found to be significant (Figs. 1,2 and Figs. 1,2 Suppl.).

Graphene oxide enhanced or inhibited seed germination of rice. Seed germination of 'K866' increased with the increase of GO concentration. However, higher concentrations of GO suppressed seed germination of 'MH63'. Moreover, GO did not affect seed germination of 'R527' and '9311'. The 'R527' contributed the highest GI in control and GO treatments. In '9311', high GO concentrations $\left(50,100,150 \mathrm{mg} \mathrm{dm}^{-3}\right)$ promoted seed germination more than low GO concentrations ( 5 or $10 \mathrm{mg} \mathrm{dm}^{-3}$ ). Graphene oxide at 5 to $150 \mathrm{mg} \mathrm{dm}^{-3}$ enhanced seed germination of 'Nipponbare', whereas no significant difference existed among GO treatments. (Fig. $1 A$ and Fig. $1 A$ Suppl.).

The shoot elongation of all rice genotypes showed a decreasing tendency with the increase of GO concentration. AtrelativelyhighGOconcentrations $\left(50,100,150 \mathrm{mg} \mathrm{dm}^{-3}\right)$, GO treatment significantly inhibited shoot elongation of 


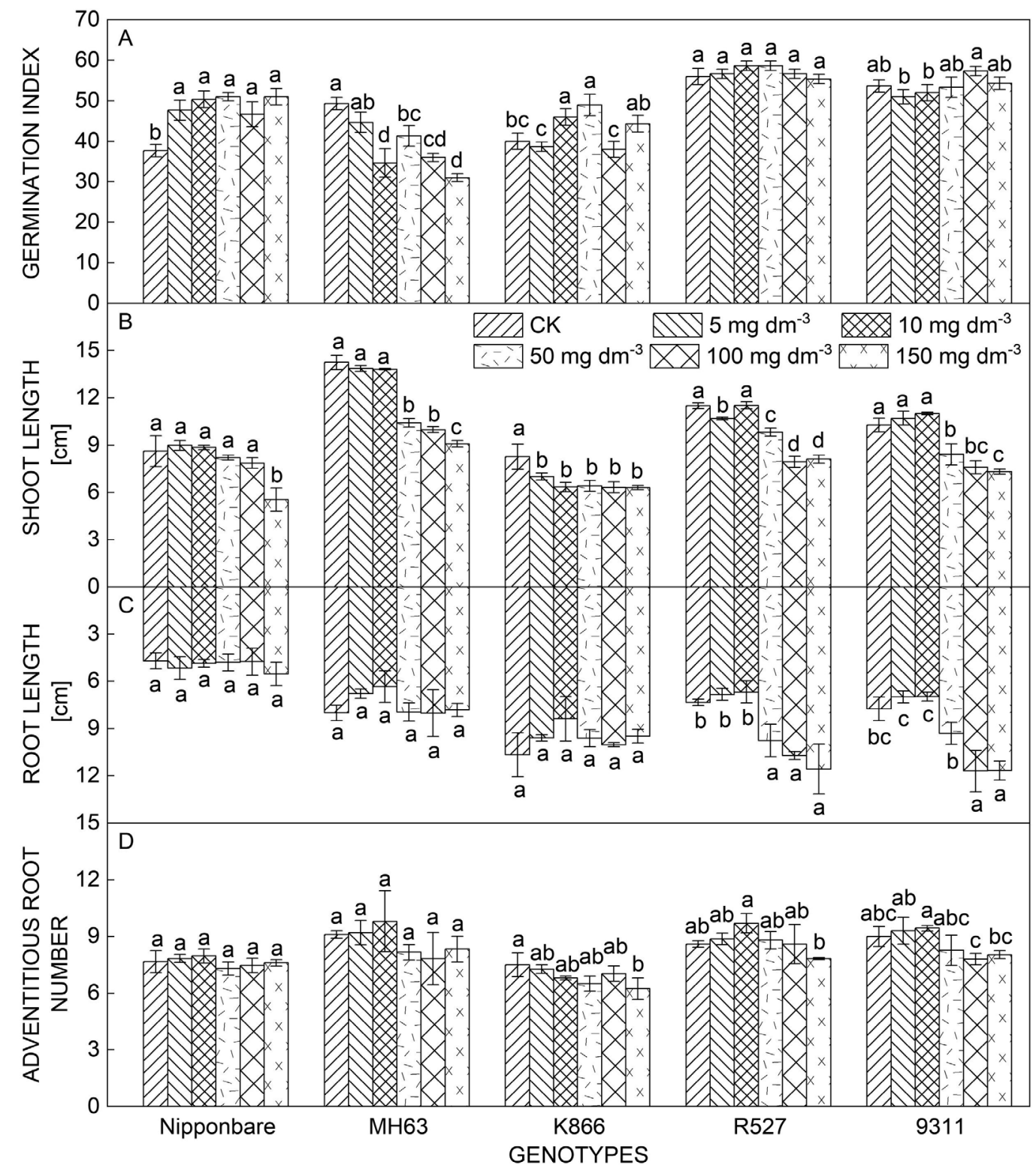

Fig. 1. Germination index $(A)$, shoot length $(B)$, root length $(C)$, and adventitious root number $(D)$ of five different rice genotypes in response to different graphene oxide (GO) dosages for $16 \mathrm{~d}$. Means $\pm \mathrm{SDs}, n=3$. Lower-case letters indicate significant differences (Duncan's multiple range test, $P<0.01$ ) between the control and GO treatments within particular genotypes.

'MH63', 'R527', and '9311'. When the GO concentration was up to $150 \mathrm{mg} \mathrm{dm}^{-3}$, the shoot elongation of 'Nipponbare' was suppressed. GO at $5 \sim 150 \mathrm{mg} \mathrm{dm}^{-3}$ inhibited the shoot elongation of 'K866', but no significant difference was found among GO treatments. The 'MH63' displayed the highest SL at the control or GO treatments, while ' $\mathrm{K} 866$ ' showed the lowest SL (Fig. $1 B$ and Fig. $1 B$ Suppl.).

Graphene oxide did not affect the root elongation of 'Nipponbare', 'MH63', and 'K866'. However, the root elongation of 'R527' and '9311' increased with the increase of GO concentration, and higher GO concentrations (50, $100,150 \mathrm{mg} \mathrm{dm}^{-3}$ ) could promote root elongation more than lower $\mathrm{GO}$ concentrations $\left(5,10 \mathrm{mg} \mathrm{dm}^{-3}\right)$. Meanwhile, 'K866' had higher RL than the other four genotypes under most GO treatments, while 'R527' and '9311' only showed higher RL at high GO concentration $\left(50,100,150 \mathrm{mg} \mathrm{dm}^{-3}\right)$. 'Nipponbare' displayed the lowest RL from all rice genotypes at GO treatments (Fig. $1 C$ and Fig. $1 C$ Suppl.).

In all rice genotypes, the ARN showed no significant response to GO. Compared with the control, only GO at $150 \mathrm{mg} \mathrm{dm}^{-3}$ significantly reduced the ARN of 'K866'. Moreover, 'MH63', 'R527', and '9311' displayed higher ARN (Fig. $1 D$ and Fig. $1 D$ Suppl.).

Shoot fresh mass (SFM) of rice also showed a decreasing tendency with increasing GO concentration. In 'MH63', 'R527', and '9311', higher GO concentrations $\left(50,100,150 \mathrm{mg} \mathrm{dm}^{-3}\right)$ induced more inhibition than low GO concentrations $\left(5,10 \mathrm{mg} \mathrm{dm}^{-3}\right)$. Although GO did not affect the SFM of 'Nipponbare' and 'K866', a significantly decreased SFM could be observed at all GO treatments 


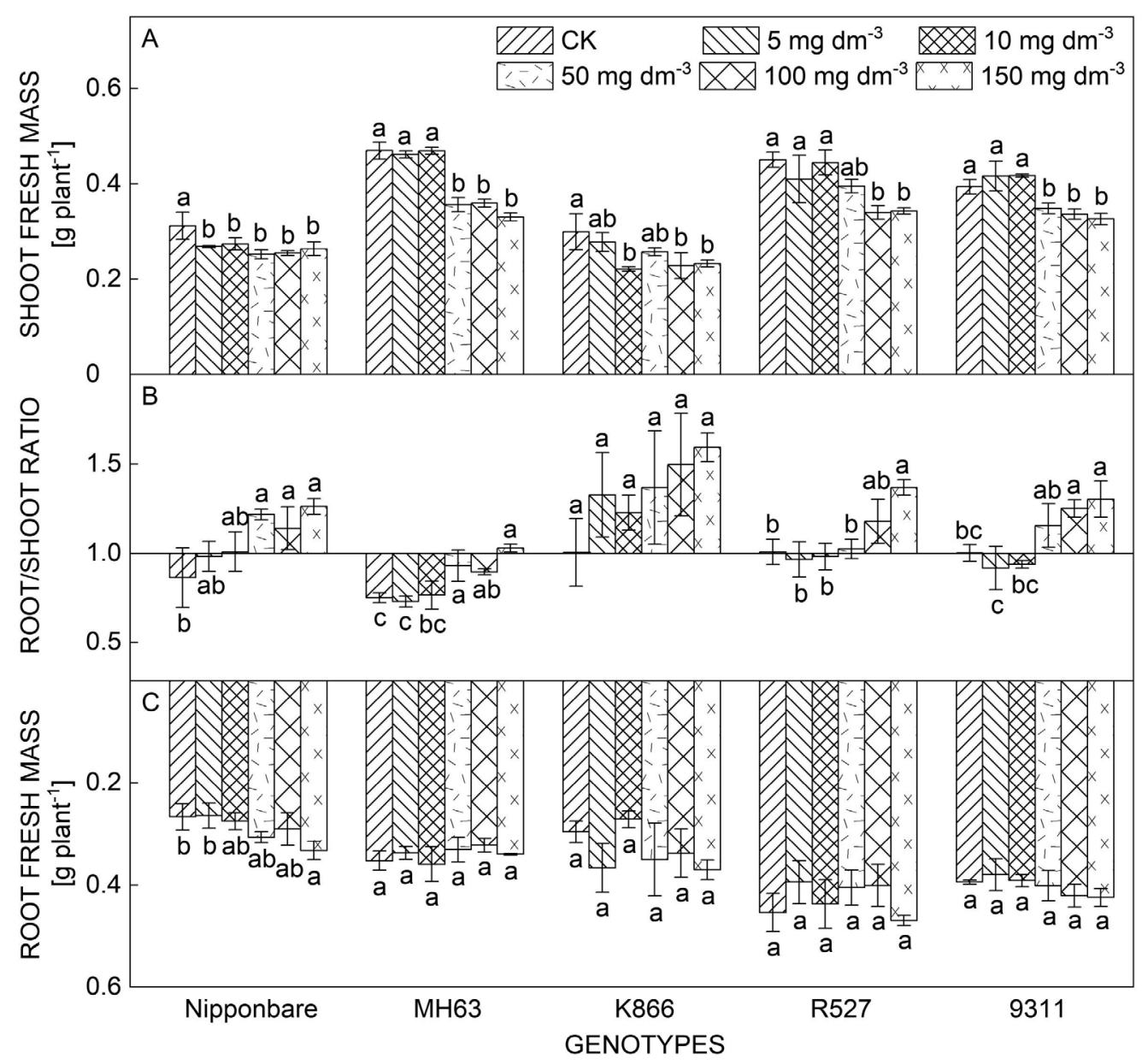

Fig. 2. Shoot $(A)$ and root $(C)$ fresh masses, and root/shoot ratio $(B)$ of five different rice genotypes in response to different graphene oxide (GO) dosages for $16 \mathrm{~d}$. Means $\pm \mathrm{SDs}, n=3$. Lower-case letters indicate significant differences (Duncan's multiple range test, $P<$ $0.01)$ between the control and GO treatments within particular genotypes.

compared with the control (Fig. $2 A$ and Fig. $2 A$ Suppl.).

No significant dose-dependent response to GO treatments was found in the root fresh mass (RFM) of all rice genotypes except 'Nipponbare'. GO at $150 \mathrm{mg} \mathrm{dm}^{-3}$ significantly increased RFM of 'Nipponbare'. However, the genotype-dependent effect of GO treatments could be observed. At all GO treatments, 'R527' and '9311' displayed higher RFM than the other rice genotypes (Fig. $2 C$ and Fig. $2 C$ Suppl.).

The R/S of all rice genotypes showed an increasing trend with the increase of GO concentration except of 'K866'. GO did not affect the R/S of 'K866'. GO at 5 $150 \mathrm{mg} \mathrm{dm}^{-3}$ strengthened R/S of 'Nipponbare', while no significant difference existed among GO treatments. Enhanced R/S of 'MH63' and '9311' appeared at GO 50 $150 \mathrm{mg} \mathrm{dm}^{-3}$ Furthermore, GO at $100 \mathrm{mg} \mathrm{dm}^{-3}$ strengthened R/S of 'R527' (Fig. $2 B$ and Fig. $2 B$ Suppl.).

These results suggested that GO could stimulate seed germination, root growth, and reduce shoot growth. The effectiveness is dose-dependent and genotype-dependent.

Under control and GO treatments, 'K866' always showed the lowest $\mathrm{Chl}$ and MDA content. Graphene oxide at concentrations $50-150 \mathrm{mg} \mathrm{dm}^{-3}$ significantly reduced the Chl content in all rice genotypes except 'K866'. Graphene oxide did not impact the MDA content of all rice genotypes (Fig. 3 and Fig. 3 Suppl.).

With the increase of GO concentration, the SOD activities showed down-regulation in all rice genotypes except 'MH63', which showed up-regulation. Meanwhile, GO at $50-150 \mathrm{mg} \mathrm{dm}^{-3}$ significantly inhibited the SOD activities of 'Nipponbare', 'K866', and '9311', and enhanced that of 'MH63'. The SOD activity of 'R527' was not affected by GO (Fig. $4 A$ ).

The CAT activities of all rice genotypes were significantly up-regulated following GO treatments. GO at $10-150 \mathrm{mg} \mathrm{dm}^{-3}$ enhanced the CAT activities of rice genotypes except '9311'. High CAT activity of '9311' appeared at GO $100 \mathrm{mg} \mathrm{dm}^{-3}$, while CAT activity showed no significant difference between the control and GO treatments (Fig. 4B).

As shown in Fig. $4 C$, the POD activities of 'Nipponbare', 'R527', and '9311' showed up-regulation with the increasing GO concentration, while no significant difference was found in 'R527'. On the contrary, the POD activities of 'MH63' and 'K866' were down-regulated by the gradually increased GO concentrations, but they 


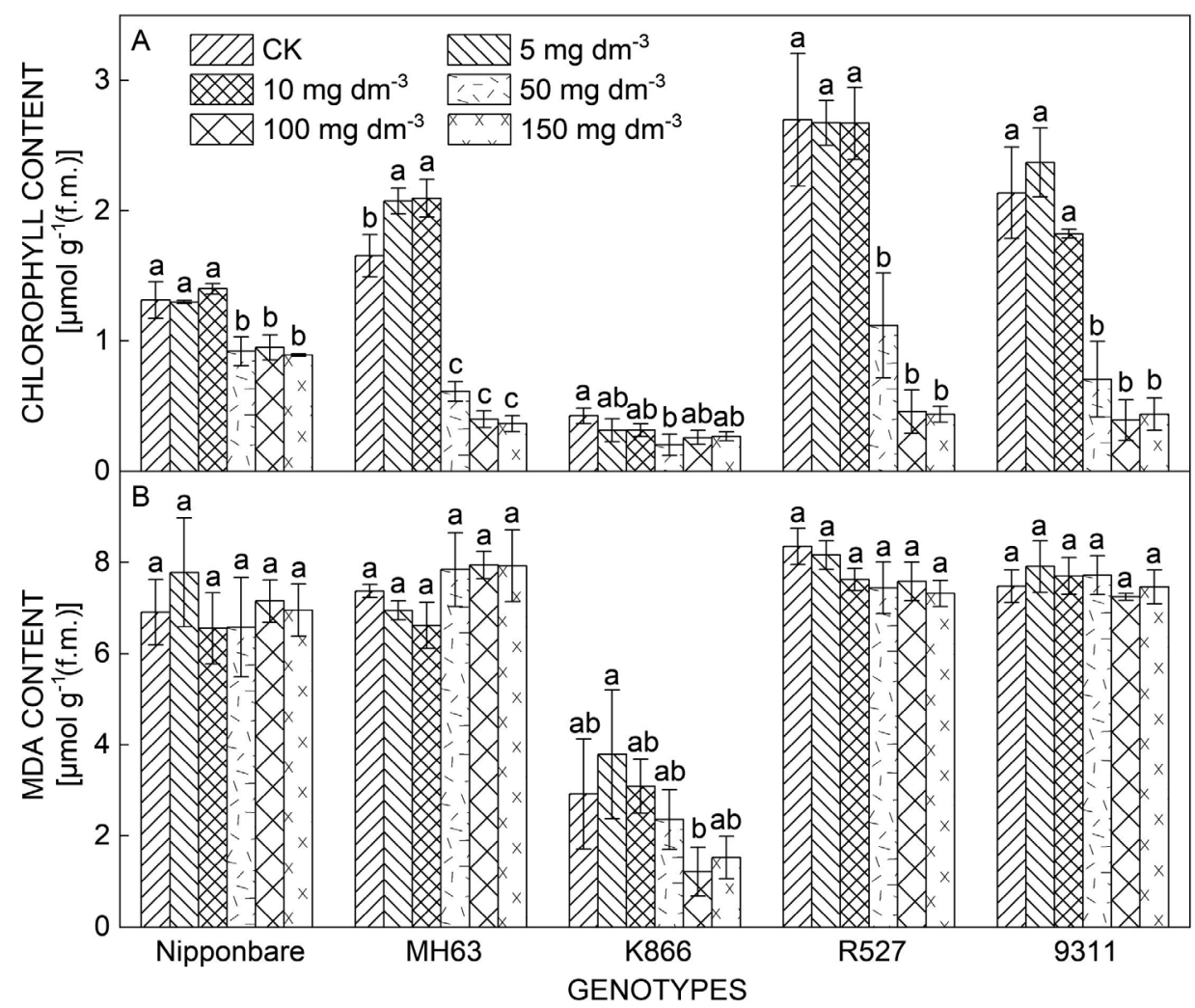

Fig. 3. Chlorophyll $(A)$ and malondialdehyde (MDA) content $(B)$ of five different rice genotypes in response to different graphene oxide (GO) dosages for $16 \mathrm{~d}$. Means $\pm \mathrm{SDs}, n=3$. Lower-case letters indicate significant differences (Duncan's multiple range test, $P<0.01$ ) between the control and GO treatments within particular genotypes.

differed only a little.

\section{Discussion}

The analysis of GI in all selected rice genotypes suggested that GO could accelerate, suppress, or not affect seeds germination of rice, and the effectiveness is genotype-dependent. Some literature reported that carbon nanomaterials could enhance seeds germination of a plant (Khodakovskaya et al. 2009, Nair et al. 2012, Zhang et al. 2015). They hypothesized that carbon nanomaterials could penetrate the seed coat, facilitate water uptake of the seed, and faster germination. However, some researchers reported the inhibition of seed germination by carbon nanomaterials (Nair et al. 2012, Liu et al. 2015, Yin et al. 2018). Nair et al. (2012) considered that micron-sized sheets of graphene blocked rice seeds coat pores, thus resulted in less water uptake and reduced germination. Yin et al. (2018) showed that $\mathrm{GO}$ induces the severe $\mathrm{H}_{2} \mathrm{O}_{2}$ production and oxidative stress in maize seeds which might suppress their germination. Lin and Xing (2007) inclined to the view that selective permeability of seed coat (Wierzbicka and Obidzińska 1998) might prevent multi-walled carbon nanotubes pass through seed coats to affect plant seed germination. In fact, most of this literature indicate that carbon nanomaterials could affect germination by adjusting water uptake of plant seed.
In our study, the genotype-dependent response of rice germination to GO indicated that the difference in seed structure might impact the interaction between GO and seed, and affect seed germination. Further investigation on the differences of seed structure will help us to explain the mechanism of GO inhibiting the germination of 'MH63' and promoting the germination of 'Nipponbare' and 'K866'. It might be helpful for us to further understand the mechanism of GO affecting plant seed germination.

Based on the comprehensive analysis of the seedling growth of rice, we found that the GO can inhibit and stimulate the shoot and root growth, respectively. Moreover, the significantly increased $\mathrm{R} / \mathrm{S}$ with the increasing GO concentration in all genotypes except 'K866' confirmed that. All selected rice genotypes adopted the same strategy to increase $\mathrm{R} / \mathrm{S}$ at different $\mathrm{GO}$ treatments. GO inhibited shoot elongation and reduced shoot biomass, and promoted root elongation and increased root biomass, while didn't affect ARN. Moreover, effectiveness is genotypedependent and dose-dependent (Figs. 1,2).

It is typical for $\mathrm{R} / \mathrm{S}$ of a plant to increase when water is limiting (Wu and Cosgrove 2000, Sinclair et al. 2005). As a response to abiotic stressors (Poeplau and Kätterer 2017), R/S reflects that plants follow distinct strategies to up-ground growth for obtaining sufficient sun radiation or down-ground growth for competition for uptake of water and nutrients (Agathokleous et al. 2019). Roots can continue elongation and shoot growth is inhibited at low 


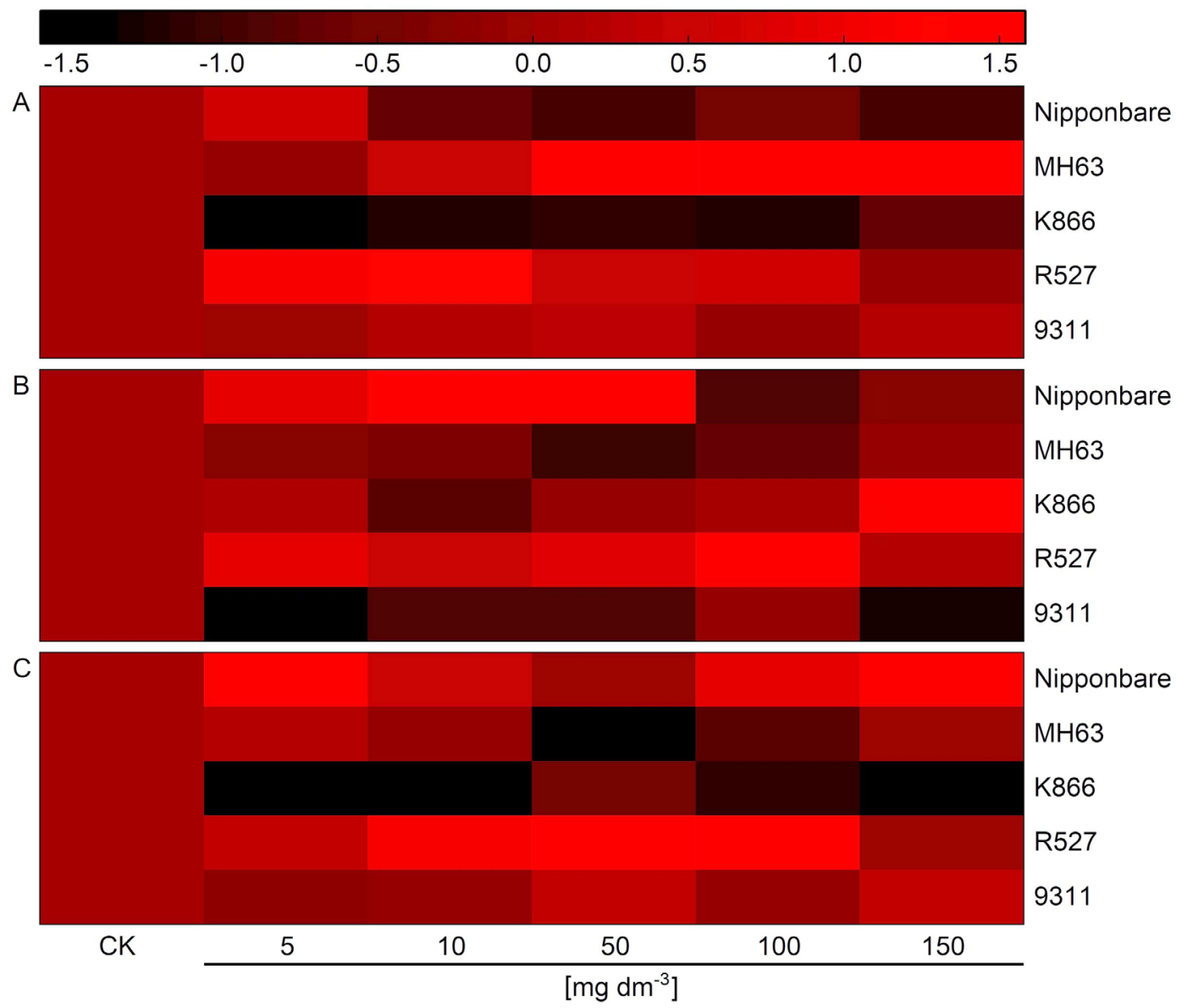

Fig. 4. A heat map of superoxide dismutase $(A)$, catalase $(B)$, and peroxidase $(C)$ activities of five different rice genotypes in response to different graphene oxide (GO) dosages for $16 \mathrm{~d}$. Relative changes are shown as an increased colour intensity according to the instance based on a difference between the control and each treatment within the particular genotype, as shown in the key.

water potentials (Nonami and Boyer 1990, Chazen and Neumann 1994). In our study, the increase of R/S showed that the water absorption capacity of rice roots might be potentially affected by GO. It is necessary to investigate the mechanism of $\mathrm{GO}$ enhancing $\mathrm{R} / \mathrm{S}$ and impacting the water uptake capacity of roots. At present, many papers suggest an explanation that carbon nanomaterials can penetrate root cells or adhese on the roots to adjust water uptake by root and affect the root growth. Cañas et al. (2008) reported that both functionalized and nonfunctionalized carbon nanotubes could form nanotube sheets on cucumber root surfaces, while not enter the root, and enhance root elongation in both onion and cucumber. Khodakovskaya et al. (2009) in tomato proved that the carbon nanomaterials are able to penetrate the root systems of plants. Miralles et al. (2014) found that the epidermis of wheat root tip could uptake of $\mathrm{Fe}_{3} \mathrm{O}_{4}$-functionalized carbon nanomaterials. Zhang et al. (2015) reported that graphene was able to penetrate root tip cells at the stage of tomato seedling growth. Zhang et al. (2020) found that GO could be absorbed on the rice root surface and make a significant change of GO morphology. Moreover, the root exudate layer between the GO and the root epidermis cells might prevent GO from entering the roots.
The mechanism of carbon nanomaterials improving water uptake of plant roots are not yet fully understood. Nevertheless, it does not prevent us from considering GO as a water-deficit stress factor when evaluating the phytotoxicity of GO.

The oxidative damage is a typical response to an abiotic stressor. An enhanced antioxidative defense system is essential to alleviate oxidative damage. The change of Chl and MDA content and antioxidant enzyme activities (SOD, CAT, and POD) in plants is usually used to monitor the possible oxidative damage (Hegedüs et al. 2001, Siddiqui et al. 2015a). Therefore, the change in Chl and MDA content and antioxidant enzymes activities in all rice genotypes showed that the oxidative damage might be occurring. Meanwhile, some researchers reported that engineered nanomaterials could conduct the changes of physio-biochemical properties and antioxidant enzyme activities (Kovacic and Somanathan 2010, Begum et al. 2014). Even though some mechanisms how abiotic stresses affect $\mathrm{Chl}$ content have been reported (Ruan et al. 2002, Panda and Khan 2009), it is not yet fully understood.

Furthermore, MDA content is an important indicator of lipid peroxidation (Hegedüs et al. 2001), which reflected the damage of cell membranes (Li et al. 2007, Siddiqui 
et al. 2015a). In our study, no significant difference of MDA content in roots was found among control and GO treatments in all genotypes except ' $\mathrm{K} 866$ ', which is similar to the results that reported by Cheng et al. (2016) and Zhang et al. (2020). Moreover, the results suggested that 'K866' suffered less damage than the other four genotypes under GO treatments.

The seedling roots of rice might more obviously indicate the toxic symptoms of pollutants than shoots due to the direct contact with nanoparticles (Sresty and Madhava Rao 1999). They would show a dose-dependent response of the root growth of sensitive plant species (Lin and Xing 2007). Meanwhile, nanoparticles could induce oxidative stress due to overproduction of reactive oxygen species in the seedling roots, which leads to potential damage to major biological macromolecules including saccharides, lipids, nucleic acids, and proteins (Kovacic and Somanathan 2010, Begum et al. 2014). Thus, the antioxidant enzyme activities, including SOD, CAT, and POD, could indirectly indicate the response of the plant to GO. Our correlation analysis (Fig. 4 Suppl.) of rice seedling growth with the antioxidant enzyme activities indicated that CAT contributed more than SOD and POD to rice seedling growth changes. It is inconsistent with the result that plant growth could be regulated by POD, which participated in the biosynthesis of lignin and reduced the plasticity of cell walls (Gajewska and Skłodowska 2007). In our study, we found no obvious changes in MDA, SOD, $\mathrm{CAT}$, and POD in rice roots. The studies done by Zhang et al. (2020) in rice also proved that GO and reduced GO did not conduct changes of SOD, POD, and MDA in roots. Moreover, they described a new mechanism of nanomaterial phytotoxicity by induced $\mathrm{pH}$ alteration, iron overload, and subsequent oxidative damage in rice.

\section{Conclusions}

Based on the comprehensive analysis of germination, seedling morphology, Chl and MDA content, and antioxidant enzyme activities, we considered that the responses of five rice genotypes to GO exhibited genotypic variation. 'R527' should be a high tolerant genotype to GO, while 'MH63' and 'K866' were more sensitive than 'Nipponbare' and '9311'. Meanwhile, the enhanced seeds germination and root growth, and the inhibited shoot growth of all five genotypes, were due to the increased antioxidant enzyme activities, especially CAT. It indicated that the GOtolerant genotype might avoid damage caused by GO by the increased antioxidant enzyme activities. Moreover, we should consider the genotype differences in the potential GO phytotoxicity and confirm the potential environmental risk of GO to ecosystems. These results would contribute to the objective evaluation of phytotoxicity of carbonbased engineered nanomaterials including GO, and further investigate the potential mechanism of tolerance in rice to GO can be important.

\section{References}

Abei, H.: Catalase in vitro. - Methods Enzymol. 105: 121-126, 1984.

Agathokleous, E., Belz, R.G., Kitao, M., Koike, T., Calabrese, E.J.: Does the root to shoot ratio show a hormetic response to stress? An ecological and environmental perspective. - J. Forest. Res. 30: 1569-1580, 2019.

Arnon, D.I.: Copper enzymes in isolated chloroplasts, polyphenol oxidase in Beta vulgaris. - Plant Physiol. 24: 1-15, 1949.

Begum, P., Ikhtiari, R., Fugetsu, B.: Potential impact of multiwalled carbon nanotubes exposure to the seedling stage of selected plant species. - Nanomaterials 4: 203-221, 2014.

Cakmak, I., Marschner, H.: Magnesium deficiency and high light intensity enhance activities of superoxide dismutase, ascorbate peroxidase, and glutathione reductase in bean leaves. - Plant Physiol. 98: 1222-1227, 1992.

Cañas, J.E., Long, M., Nations, S., Vadan, R., Dai, L., Luo, M.X., Ambikapathi, R., Lee, E.H., Olszyk, D.: Effects of functionalized and nonfunctionalized single-walled carbon nanotubes on root elongation of select crop species. - Environ. Toxicol. Chem. 27: 1922-1931, 2008.

Chazen, O., Neumann, P.M.: Hydraulic signals from the roots and rapid cell-wall hardening in growing maize (Zea mays L.) leaves are primary responses to polyethylene glycol- induced water deficits. - Plant Physiol. 104: 1385-1392, 1994.

Chen, L., Wang, C., Li, H., Qu, X., Yang, S.T., Chang, X.L.: Bioaccumulation and toxicity of ${ }^{13} \mathrm{C}$-skeleton labeled graphene oxide in wheat. - Environ. Sci. Technol. 51: 1014610153, 2017.

Cheng, F., Liu, Y.F., Lu, G.Y., Zhang, X.K., Xie, L.L., Yuan, C.F., Xu, B.B.: Graphene oxide modulates root growth of Brassica napus L. and regulates ABA and IAA concentration. - J. Plant Physiol. 193: 57-63, 2016.

Gajewska, E., Skłodowska, M.: Effect of nickel on ROS content and antioxidative enzyme activities in wheat leaves. BioMetals. 20: 27-36, 2007.

Hao, Y., Ma, C., Zhang, Z., Song, Y., Cao, W., Guo, J., Zhou, G., Rui, Y., Liu, L., Xing, B.: Carbon nanomaterials alter plant physiology and soil bacterial community composition in a rice-soil-bacterial ecosystem. - Environ. Pollut. 232: 123-136, 2018.

Heath, R.L., Packer, L.: Photoperoxidation in isolated chloroplasts. I. Kinetics and stoichiometry of fatty acid peroxidation. - Arch. Biochem. Biophys. 125: 189-198, 1968.

Hegedüs, A., Erdei, S., Horváth, G.: Comparative studies of $\mathrm{H}_{2} \mathrm{O}_{2}$ detoxifying enzymes in green and greening barley seedlings under cadmium stress. - Plant Sci. 160: 1085-1093, 2001.

Huang, Q., Liu, Q., Lin, L., Li, F.J., Han, Y., Song, Z.G.: Reduction of arsenic toxicity in two rice cultivar seedlings by different nanoparticles. - Ecotoxicol. environ. Safety 159: 261-271, 2018.

Jiao, J., Cheng, F., Zhang, X., Xie, L., Li, Z., Yuan, C., Xu, B., Zhang, L.: Preparation of graphene oxide and its mechanism in promoting tomato roots growth. - J. Nanosci. Nanotechnol. 16: 4216-4223, 2016a.

Jiao, J., Yuan, C., Wang, J., Xia, Z., Xie, L., Chen, F., Li, Z., Xu, B.: The role of graphene oxide on tobacco root growth and its preliminary mechanism. - J. Nanosci. Nanotechnol. 16: 12449-12454, $2016 \mathrm{~b}$.

Keller, A.A., Lazareva, A.: Predicted releases of engineered nanomaterials: from global to regional to local. - Environ. Sci. Technol. Lett. 1: 65-70, 2013.

Khodakovskaya, M., Dervishi, E., Mahmood, M., Xu, Y., Li, Z., Watanabe, F., Biris, A.S.: Carbon nanotubes are able to penetrate plant seed coat and dramatically affect seed 
germination and plant growth. - ACS Nano. 3: 3221-3227, 2009.

Kovacic, P., Somanathan, R.: Biomechanisms of nanoparticles (toxicants, antioxidants and therapeutics): electron transfer and reactive oxygen species. - J. Nanosci. Nanotechnol. 10: 7919-7930, 2010.

Li, C.X., Feng, S.L., Shao, Y., Jiang, L.N., Lu, X.Y., Hou, X.L.: Effects of arsenic on seed germination and physiological activities of wheat seedlings. - J. environ. Sci. (China) 19: 725-732, 2007.

Lin, D.H., Xing, B.S.: Phytotoxicity of nanoparticles: inhibition of seed germination and root growth. - Environ. Pollut. 150: 243-250, 2007.

Liu, F., Ming, P., Li, J.: Ab initio calculation of ideal strength and phonon instability of graphene under tension. - Phys. Rev. B 76: 471-478, 2007.

Liu, S., Wei, H., Li, Z., Li, S., Yan, H., He, Y., Tian, Z.: Effects of graphene on germination and seedling morphology in rice. - J. Nanosci. Nanotechnol. 15: 2695-2701, 2015.

Miralles, P., Johnson, E., Church, T.L., Harris, A.T.: Multiwalled carbon nanotubes in alfalfa and wheat: toxicology and uptake. - J. R. Soc. Interface 9: 3514-3527, 2012.

Nair, R., Mohamed, M.S., Gao, W., Maekawa, T., Yoshida, Y., Ajayan, P.M., Kumar, D.S.: Effect of carbon nanomaterials on the germination and growth of rice plants. - J. Nanosci. Nanotechnol. 12: 2212-2220, 2012.

Nonami, H., Boyer, J.S.: Primary events regulating stem growth at low water potentials. - Plant Physiol. 93: 1601-1609, 1990.

Panda, S.K., Khan, M.H.: Growth, oxidative damage and antioxidant responses in greengram (Vigna radiata L.) under short-term salinity stress and its recovery. - J. Agron. Crop Sci. 195: 442-454, 2009.

Poeplau, C., Kätterer, T.: Is soil texture a major controlling factor of root:shoot ratio in cereals?. - Eur. J. Soil Sci. 68: 964-970, 2017.

Ruan, H., Shen, W., Ye, M., Xu, L.: Protective effects of nitric oxide on salt stress-induced oxidative damage to wheat (Triticum aestivum L.) leaves. - Chin Sci. Bull. 47: 677-681, 2002.

Siddiqui, M.H., Al-Khaishany, M.Y., Al-Qutami, M.A., AlWhaibi, M.H., Grover, A., Ali, H.M., Al-Wahibi, M.S.: Morphological and physiological characterization of different genotypes of faba bean under heat stress. - Saudi J. biol. Sci. 22: 656-663, 2015a.

Siddiqui, M.H., Al-Khaishany, M.Y., Al-Qutami, M.A., AlWhaibi, M.H., Grover, A., Ali, H.M., Al-Wahibi, M.S.,
Bukhari, N.A.: Response of different genotypes of faba bean plant to drought stress. - Int. J. mol. Sci. 16: 10214-10227, 2015 b.

Sinclair, T.R., Holbrook, N.M., Zwieniecki, M.A.: Daily transpiration rates of woody species on drying soil. - Tree Physiol. 25: 1469-1472, 2005.

Singh, V., Joung, D., Lei, Z., Das, S., Khondaker, S.I., Seal, S.: Graphene based materials: past, present and future. - Progr. Mater. Sci. 56: 1178-1271, 2011.

Sresty, T.V.S., Madhava Rao, K.V.: Ultrastructural alterations in response to zinc and nickel stress in the root cells of pigeonpea. - Environ. exp. Bot. 41: 3-13, 1999.

Tribouillois, H., Durr, C., Demilly, D., Wagner, M.H., Justes, E.: Determination of germination response to temperature and water potential for a wide range of cover crop species and related functional groups. - PLoS ONE 11: e0161185, 2016.

Wang, C., Xiao, H., Zhao, L., Liu, J., Wang, L., Zhang, F., Shi, Y., Du, D.: The allelopathic effects of invasive plant Solidago canadensis on seed germination and growth of Lactuca sativa enhanced by different types of acid deposition. Ecotoxicology 25: 555-562, 2016.

Wierzbicka, M., Obidzińska, J.: The effect of lead on seed imbibition and germination in different plant species. - Plant Sci. 137: 155-171, 1998.

Wu, Y., Cosgrove, D.J.: Adaptation of roots to low water potentials by changes in cell wall extensibility and cell wall proteins. - J. exp. Bot. 51: 1543-1553, 2000.

$\mathrm{Xu}$, L., Duan, L., Chen, W.: Carbon nanomaterials: their environmental behavior and effects on the transport and fate of pollutants in environment. - Chin. J. appl. Ecol. 20: 870$875,2009$.

Yin, L.Y., Wang, Z., Wang, S.G., Xu W.Y., Bao H.F.: Effects of graphene oxide and/or $\mathrm{Cd}^{2+}$ on seed germination, seedling growth, and uptake to $\mathrm{Cd}^{2+}$ in solution culture. - Water Air Soil Pollut. 229: 151, 2018.

Zhang, M., Gao, B., Chen, J., Li, Y.: Effects of graphene on seed germination and seedling growth. - J. Nanopart. Res. 17: 78, 2015.

Zhang, P., Guo Z., Luo W.: Graphene oxide induced pH alteration, iron overload and subsequent oxidative damage in rice (Oryza. sativa L.): a new mechanism of nanomaterial phytotoxicity. - Environ. Sci. Technol. 54: 3181-3190, 2020.

Zhou, Q., Hu, X.: Systemic stress and recovery patterns of rice roots in response to graphene oxide nanosheets. - Environ. Sci. Technol. 51: 2022-2030, 2017. 